\title{
Heterologous and High Production of Ergothioneine in Escherichia coli
}

\author{
Ryo Osawa, ${ }^{\dagger}$ Tomoyuki Kamide, ${ }^{\dagger}$ Yasuharu Satoh, ${ }^{*}, \oplus$ Yusuke Kawano, ${ }^{\S}$ Iwao Ohtsu, ${ }^{\S}$ \\ and Tohru Dairi* ${ }^{*} \ddagger(0)$ \\ ${ }^{\dagger}$ Graduate School of Chemical Science and Engineering and ${ }^{\ddagger}$ Graduate School of Engineering, Hokkaido University, Sapporo, \\ Hokkaido 060-8628, Japan \\ ${ }^{\S}$ Innovation Medical Research Institute, University of Tsukuba, Tsukuba, Ibaraki 305-8550, Japan
}

\section{Supporting Information}

ABSTRACT: Ergothioneine (ERG) is a histidine-derived thiol compound suggested to function as an antioxidant and cytoprotectant in humans. Therefore, experimental trials have been conducted applying ERG from mushrooms in dietary supplements and as a cosmetic additive. However, this method of producing ERG is expensive; therefore, alternative methods for ERG supply are required. Five Mycobacterium smegmatis genes, egtABCDE, have been confirmed to be responsible for ERG biosynthesis. This enabled us to develop practical fermentative ERG production by microorganisms. In this study, we carried out heterologous and high-level production of ERG in Escherichia coli using the egt genes from M. smegmatis. By high production of each of the Egt enzymes and elimination of bottlenecks in the substrate supply, we succeeded in constructing a production system that yielded $24 \mathrm{mg} / \mathrm{L}(104 \mu \mathrm{M})$ secreted ERG.

KEYWORDS: ergothioneine, heterologous production, Escherichia coli

\section{INTRODUCTION}

Ergothioneine (ERG), a histidine-derived thiol compound, was isolated from an ergot fungus, Claviceps purpurea, more than a century ago. ERG is also known to be synthesized in actinobacteria, cyanobacteria, and a fission yeast. ${ }^{1-3}$ Recent studies show that ERG functions as an antioxidant, such as glutathione, mycothiol, and bacillithiol. There is no direct evidence for biosynthesis of ERG in humans. However, ERG has been reported to be accumulated in various cells and tissues at high concentrations, probably by intake from diets, such as mushrooms and red beans, that contain relatively large amounts of ERG through an ERG-specific organic cation transporter, OCTN1. ${ }^{4,5}$

The presence of the ERG-specific transporter and the extensive accumulation of ERG in tissues suggest that ERG should have significant biological functions in humans. Although the true physiological role of ERG in humans has yet to be fully understood, ERG has been shown by in vitro experiments to function as an antioxidant and a cytoprotectant. Therefore, applications of ERG in dietary supplements and as a cosmetic additive have been explored, and there is an increasing demand for ERG. ${ }^{6}$ Mushrooms have traditionally been the source of ERG. ${ }^{7}$ However, slow growth, low content, and timeconsuming purification procedures lead to a high manufacturing cost. Therefore, alternative and sustainable sources of ERG are necessary.

One such reliable and practical method is a fermentative process using microorganisms, such as actinobacteria and cyanobacteria, that are known to produce ERG. However, their ERG productivities are very low $(1.18 \mathrm{mg} / \mathrm{g}$ of dry weight after 4 weeks of cultivation of Mycobacterium avium and $0.8 \mathrm{mg} / \mathrm{g}$ of dry weight of Oscillatoria sp.), ${ }^{2,8}$ and thus, genetic and metabolic engineering are indispensable for industrial production. Until recently, however, there were no reports on ERG biosynthesis genes and enzymes. In 2010, five genes in Mycobacterium smegmatis, egt $A B C D E$, were confirmed to be responsible for ERG biosynthesis (Figure 1). ${ }^{9}$ In the biosynthetic pathway, EgtD catalyzes the formation of hercynine (HER) by transfer of three methyl groups derived from $S$-adenosylmethionine (SAM) to L-histidine (L-His). Then, EgtB catalyzes $\mathrm{O}_{2}$-dependent $\mathrm{C}-\mathrm{S}$ bond formation between $\gamma$-glutamylcysteine ( $\gamma \mathrm{GC})$ supplied by EgtA and HER to form hercynyl- $\gamma$-glutamylcysteine sulfoxide ( $\gamma$ GC-HER). This is followed by removal of the L-glutamate (L-Glu) moiety by $\mathrm{EgtC}$, an amidohydrolase, to produce hercynylcysteine sulfoxide (Cys-HER). Then, EgtE, a PLP-dependent C-S lyase, catalyzes the formation of ERG with concomitant formation of pyruvate and ammonia as side products.

In this study, we developed heterologous and high-level production of ERG in Escherichia coli using the egt genes from M. smegmatis. By high production of each of the Egt enzymes and elimination of bottlenecks in substrate supply, the production system yielded $24 \mathrm{mg} / \mathrm{L}(104 \mu \mathrm{M})$ secreted ERG.

\section{MATERIALS AND METHODS}

General Procedures. Lysogeny broth (LB, Lennox) medium was purchased from Sigma-Aldrich Japan K.K. (Tokyo, Japan); Lmethionine (L-Met) and L-His were obtained from Wako Pure Chemical Industry (Osaka, Japan); and HER was purchased from Shinsei Chemical Company, Ltd. (Osaka, Japan). Other chemicals

Received: October 24, 2017

Revised: December 22, 2017

Accepted: December 25, 2017

Published: December 25, 2017 


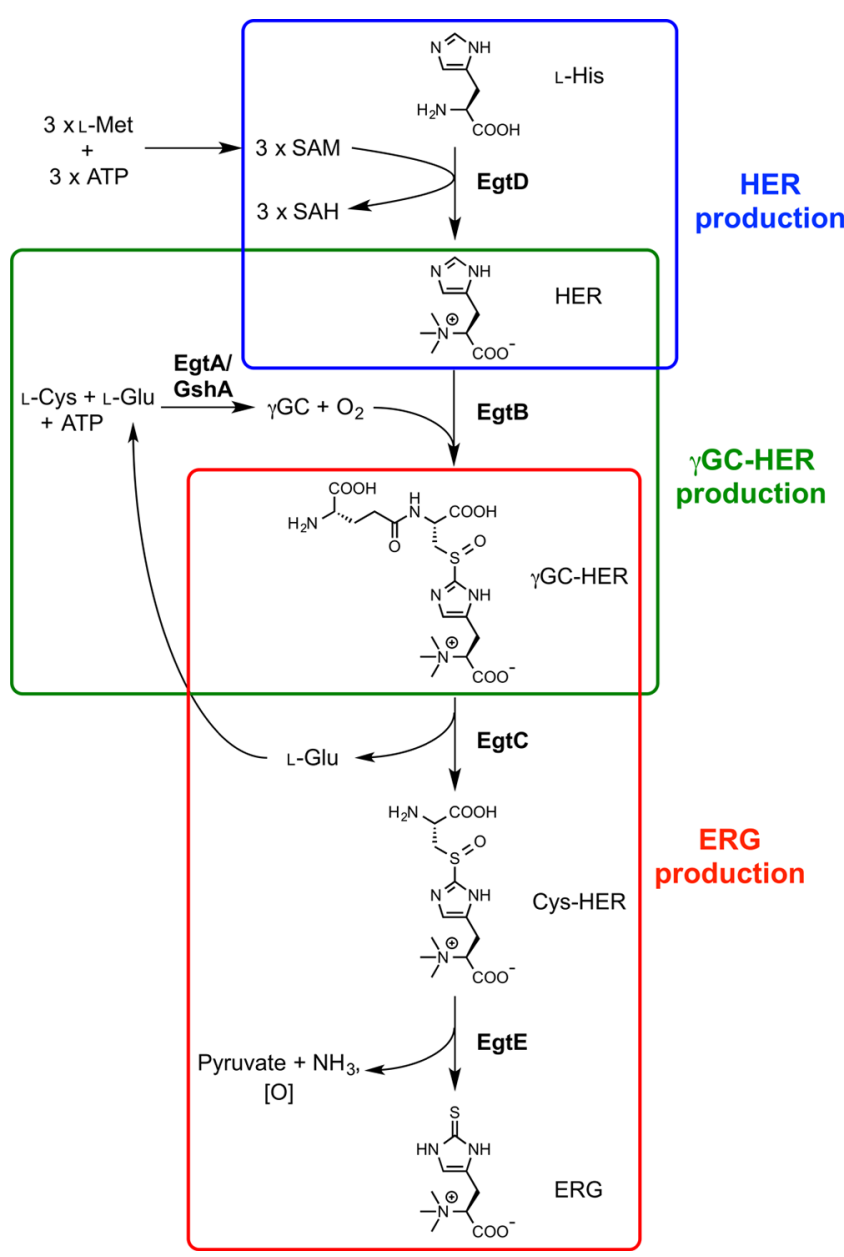

Figure 1. ERG biosynthetic pathway.

were of analytical grade and purchased from Wako Pure Chemical Industry or Sigma-Aldrich Japan. Primers were obtained from FASMAC Co., Ltd. (Kanagawa, Japan). Enzymes and kits for DNA manipulation were purchased from Takara Bio, Inc. (Shiga, Japan) or New England BioLabs Japan, Inc. (Tokyo, Japan). Polymerase chain reaction (PCR) was carried out using a GeneAmp PCR System 9700 thermal cycler (Thermo Fisher Scientific, Inc., Waltham, MA, U.S.A.) with Tks Gflex DNA polymerase (Takara Bio). General genetic manipulations of E. coli were performed according to standard protocols. High-resolution electrospray ionization Fourier transform mass spectrometry (HR-ESI-FT-MS) analysis was performed using an Exactive system (Thermo Fisher Scientific, Inc.).

Bacterial Strains and Cultures. Microorganisms used in this study are summarized in Table 1. E. coli XL1-Blue (Nippon Gene Co., Ltd., Tokyo, Japan), BL21(DE3) (Merck KGaA, Darmstadt, Germany), and BW25113 (National Institute of Genetics, Shizuoka, Japan), which is a high producer of $\mathrm{L}$-cysteine ( $\mathrm{L}$-Cys) used for a sulfur donor of $E R G,{ }^{10}$ were used for plasmid construction, protein production, and ERG production, respectively. The media used were LB and M9Y minimal medium prepared by adding $1 \%(\mathrm{w} / \mathrm{v})$ glucose, $5 \mathrm{mM} \mathrm{MgSO}_{4}, 0.1 \mathrm{mM} \mathrm{CaCl}_{2}$, and $0.1 \%$ (w/v) yeast extract (Becton, Dickinson and Company, Franklin Lakes, NJ, U.S.A.) to M9 minimal salts (Becton, Dickinson and Company). Ampicillin (Ap), chloramphenicol $(\mathrm{Cm})$, kanamycin $(\mathrm{Km})$, streptomycin $(\mathrm{Sm})$, and tetracycline $(\mathrm{Tc})$ were added to the media at concentrations of 100 , $33,25,20$, and $5 \mathrm{mg} / \mathrm{L}$, if necessary. Optical density (OD) at $600 \mathrm{~nm}$ was measured with a NanoDrop 2000C spectrophotometer (Thermo Fisher Scientific, Inc.).

Preparation of Egt Recombinant Enzymes. Detailed plasmid construction methods are described in Supplementary Methods 1 of the Supporting Information, and the plasmids are summarized in
Table 1. Bacterial Strains Used in This Study

$\begin{array}{lrr}\text { strain } & \text { description } & \text { source } \\ \begin{array}{c}\text { M. smegmatis } \\ \text { JCM6386 }\end{array} & \text { ERG producer } & \mathrm{JCM}^{a}\end{array}$

JCM6386

E. coli

\begin{tabular}{|c|c|c|}
\hline XL1-Blue & $\begin{array}{l}\text { hs } d R 17 \text {, recA } 1 \text {, end } 1, \text { gyrA96, thi- } 1 \text {, } \\
\text { supE44, relA } 1, \operatorname{lac}\left[\mathrm{F}^{\prime}, \text { proAB, }\right. \\
\left.\text { lac } I^{q} \mathrm{Z} \Delta \mathrm{M} 15, \operatorname{Tn} 10\left(\operatorname{Tc}^{R}\right)\right]\end{array}$ & Nippon Gen \\
\hline BL21(DE3) & 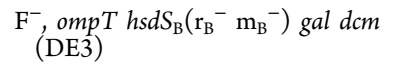 & Merck \\
\hline BW25113 & $\begin{array}{l}r r n B 3 \Delta l a c Z 4787 h s d R 514 \\
\quad \Delta(\operatorname{araBAD}) 567 \Delta(\text { rhaBAD)568 } \\
\quad r p h-1\end{array}$ & $\mathrm{NIG}^{b}$ \\
\hline ET1 & BW25113 harboring pCF1s-MsD & this study \\
\hline ET2 & $\begin{array}{l}\text { BW25113 harboring pCF1s-MsD, } \\
\text { pQE1a-mMsB }\end{array}$ & this study \\
\hline ET3 & $\begin{array}{l}\text { BW25113 harboring pCF1s-MsD, } \\
\text { pQE1a-mMsB, } \\
\text { pAC1c-hMsC/hMsE }\end{array}$ & this study \\
\hline ET4 & $\begin{array}{l}\text { BW25113 harboring pCF1s-MsD, } \\
\text { pQE1a-mMsB/EcA, } \\
\text { pAC1c-hMsC/hMsE }\end{array}$ & this study \\
\hline
\end{tabular}

${ }^{a}$ Japan Collection of Microorganisms, Riken BioResource Center. ${ }^{b}$ National Institute of Genetics.

Table 2. Briefly, EgtB, EgtC, EgtD, and EgtE were amplified by PCR using M. smegmatis genomic DNA as the template and appropriate

Table 2. Plasmids Used in This Study

\begin{tabular}{|c|c|c|}
\hline plasmid & description & source \\
\hline pQEla-Red & $\begin{array}{l}\text { protein production plasmid, tac promoter, } \\
\text { ColE1 ori, } \mathrm{Ap}^{\mathrm{R}}\end{array}$ & $\begin{array}{l}\text { lab } \\
\text { stock }\end{array}$ \\
\hline pCF1s-Red & $\begin{array}{l}\text { protein production plasmid, tac promoter, } \\
\text { CDF ori, } \mathrm{Sm}^{\mathrm{R}}\end{array}$ & $\begin{array}{l}\text { lab } \\
\text { stock }\end{array}$ \\
\hline pET-21a & $\begin{array}{l}\text { protein production plasmid, } T 7 \text { promoter, } \\
\text { pBR322 ori, } A^{R}\end{array}$ & Merck \\
\hline pACYCDuet-1 & $\begin{array}{l}\text { protein production plasmid, } T 7 \text { promoter, } \\
\text { p15A ori, } \mathrm{Cm}^{\mathrm{R}}\end{array}$ & Merck \\
\hline pQE1a-MsB & pQE1a-Red derivative, production of EgtB & $\begin{array}{l}\text { this } \\
\text { study }\end{array}$ \\
\hline pQE1a-mMsB & $\begin{array}{l}\text { pQE1a-MsB derivative, production of } \\
\text { MBP-fused EgtB }\end{array}$ & $\begin{array}{l}\text { this } \\
\text { study }\end{array}$ \\
\hline pQE1a-mMsB/EcA & $\begin{array}{l}\text { pQE1a-mMsB derivative, co-production } \\
\text { of MBP-fused EgtB and GshA }\end{array}$ & $\begin{array}{l}\text { this } \\
\text { study }\end{array}$ \\
\hline pQE1a-MsC & $\begin{array}{l}\text { pQE1a-Red derivative, production of } \\
\text { EgtC }\end{array}$ & $\begin{array}{l}\text { this } \\
\text { study }\end{array}$ \\
\hline pQE1a-hMsC & $\begin{array}{l}\mathrm{pQE} 1 \mathrm{a}-\mathrm{MsC} \text { derivative, production of } \\
\text { His-tagged EgtC }\end{array}$ & this \\
\hline pQE1a-MsE & pQE1a-Red derivative, production of EgtE & $\begin{array}{l}\text { this } \\
\text { study }\end{array}$ \\
\hline pQE1a-hMsE & $\begin{array}{l}\text { pQE1a-Red derivative, production of } \\
\text { His-tagged EgtE }\end{array}$ & $\begin{array}{l}\text { this } \\
\text { study }\end{array}$ \\
\hline $\mathrm{pAC} 1 \mathrm{c}-\mathrm{hMsC} / \mathrm{hMsE}$ & $\begin{array}{l}\text { pACYCDuet-1 derivative, co-production } \\
\text { of His-tagged EgtC and EgtE }\end{array}$ & $\begin{array}{l}\text { this } \\
\text { study }\end{array}$ \\
\hline pCF1s-MsD & pCF1s-Red derivative, production of EgtD & $\begin{array}{l}\text { this } \\
\text { study }\end{array}$ \\
\hline
\end{tabular}

primers (Table S1 of the Supporting Information), in which restriction sites were introduced at the $\mathrm{N}$ and $\mathrm{C}$ termini. The PCR products were respectively cloned into the expression vectors. The plasmids obtained were introduced into E. coli BL21(DE3). A liquid culture of the transformant in LB supplied with appropriate antibiotics was induced by adding $0.5 \mathrm{mM}$ isopropyl $\beta$-D-1-thiogalactopyranoside (IPTG) when the optical density at $600 \mathrm{~nm}$ reached about 0.6 . The cultivation was continued for an additional $16 \mathrm{~h}$ at $20^{\circ} \mathrm{C}$. The production of each recombinant enzyme was analyzed by sodium dodecyl sulfate polyacrylamide gel electrophoresis (SDS-PAGE) with Coomassie Brilliant Blue staining. The purification of each recombinant protein was carried out using nickel-nitrilotriacetic acid (Ni-NTA) agarose 


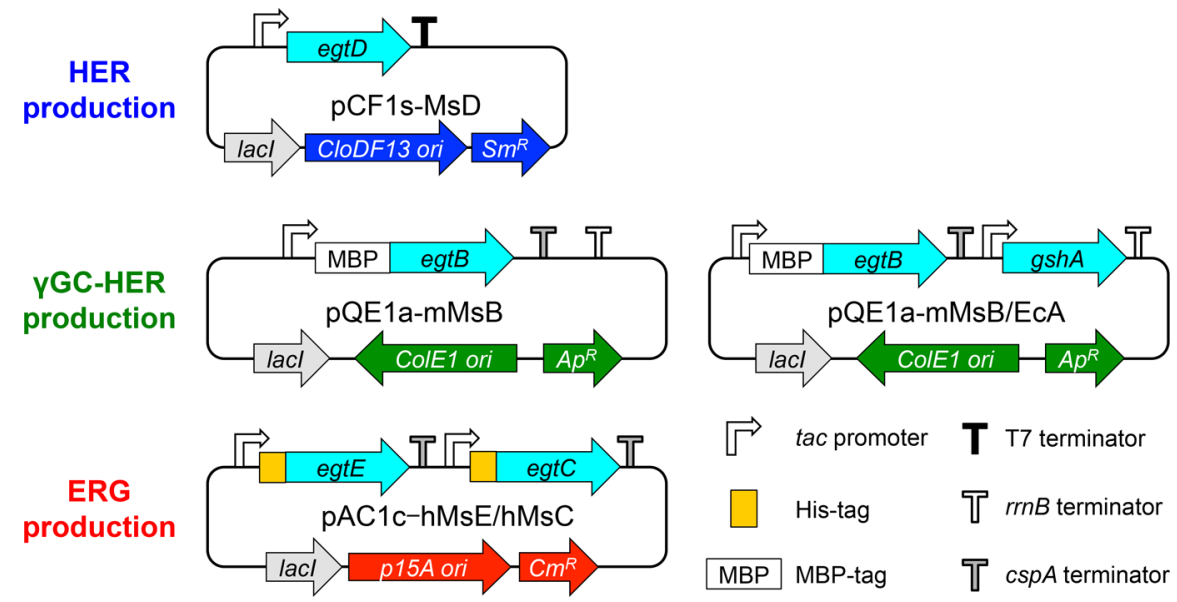

Figure 2. Plasmids for ERG production.

(QIAGEN K.K., Tokyo, Japan) or amylose resins (New England BioLabs) according to the protocols of the manufacturer. Protein concentrations were determined using a Bradford protein assay kit (Bio-Rad Laboratories, Inc., Hercules, CA, U.S.A.) with bovine serum albumin as the standard.

ERG Production. E. coli harboring plasmids carrying ERG biosynthetic genes (Figure 2) was cultured in $3 \mathrm{~mL}$ of M9Y media at $30{ }^{\circ} \mathrm{C}$ for $16 \mathrm{~h}$. The cultures $(1 \mathrm{~mL})$ were inoculated into $50 \mathrm{~mL}$ of M9Y media supplemented with $0.5 \mathrm{~g} / \mathrm{L}$ L-His, $0.5 \mathrm{~g} / \mathrm{L}$ L-Met, and 20 $\mathrm{mg} / \mathrm{L} \mathrm{FeSO} \cdot 7 \mathrm{H}_{2} \mathrm{O}$ in $200 \mathrm{~mL}$ Erlenmeyer flasks and incubated at 30 ${ }^{\circ} \mathrm{C}$ with shaking (200 rpm) for up to $72 \mathrm{~h} . \mathrm{Na}_{2} \mathrm{~S}_{2} \mathrm{O}_{3}$ was added to media at $20 \mathrm{mM}$, if needed. IPTG was added to a final concentration of $0.5 \mathrm{mM}$ after $3 \mathrm{~h}$ of cultivation. Samples $(1 \mathrm{~mL})$ were collected at appropriate time points and analyzed by liquid chromatographyelectrospray ionization-mass spectrometry (LC-ESI-MS).

LC-ESI-MS Analysis of Products. Mixtures of $0.05 \%(\mathrm{v} / \mathrm{v})$ heptafluorobutyric acid (HFBA) solution $(180 \mu \mathrm{L})$ and culture broth or enzymatic reaction solutions $(20 \mu \mathrm{L})$ were analyzed. The LC-ESIMS conditions were as follows: Waters ACQUITY UPLC system equipped with a photodiode array and a SQ Detector2 (Tokyo, Japan); XBridge BEH C18 XP column (150 mm length $\times 2.0 \mathrm{~mm}$ internal diameter, $2.5 \mu \mathrm{m}$, Waters); flow rate, $0.15 \mathrm{~mL} / \mathrm{min}$; temperature, $35{ }^{\circ} \mathrm{C}$; mobile phase, water containing $0.05 \%$ HFBA and $7 \%$ methanol; injection volume, $2 \mu \mathrm{L}$; and detection, $210 \mathrm{~nm}$ for His and HER, $250 \mathrm{~nm}$ for Cys-HER and $\gamma$ GC-HER, and $258 \mathrm{~nm}$ for ERG.

\section{RESULTS AND DISCUSSION}

The most reliable strategy for high production of ERG is overproduction of each of the enzymes for ERG biosynthesis and then optimization of any inefficient biosynthetic step(s) (identified by intermediate accumulation) by molecular genetics and metabolic engineering. If no intermediates accumulate, supply of the initial substrates, L-His, L-Glu, LCys, and L-Met, should be enhanced.

ERG biosynthetic genes are present in some microorganisms, such as actinobacteria, cyanobacteria, and $\alpha$-proteobacteria. ${ }^{11}$ Among these bacteria, we selected $M$. smegmatis as the gene source, because the ERG biosynthetic pathway was first identified in this strain and the biosynthesis genes constitute an operon. ${ }^{9}$ Because egtB, egt $C$, and egtE are translationally coupled, we first tried expressing the egt genes as the operon with tac and T7 promoters. We constructed several plasmids using pQE1a-Red and pET-21a, but ERG productivities of transformants harboring the plasmids were low (less than 0.2 $\mathrm{mg} / \mathrm{L}$ ). Therefore, we overexpressed egt genes stepwise under the control of individual promoters by checking production of recombinant enzymes and their activities by production of intermediate compounds (HER, $\gamma \mathrm{GC}-\mathrm{HER}$, and Cys-HER) and ERG by in vitro experiment (Figure 1). Because E. coli has an egt $A$ orthologue, gsh $A$, which is responsible for glutathione biosynthesis, $^{12}$ we expressed the other genes, egt $B$, egt $C$, egt $D$, and egtE. We also prepared the intermediate compounds, which are not commercially available, with the recombinant enzymes to obtain standards for quantitative analysis (Supplementary Methods 2 of the Supporting Information).

Overproduction of Recombinant Enzymes. For overproduction of Egt enzymes, we used pQE1a-Red and pCF1sRed, both of which are home-constructed and compatible vectors with the tac promoter for protein production (Figure S1 of the Supporting Information). As Figure S2 of the Supporting Information shows, transformants harboring pCF1s-MsD carrying egtD overproduced EgtD in soluble form. To confirm whether the recombinant protein had the expected activity, we carried out in vitro experiments with cell-free extracts containing the recombinant enzyme. ${ }^{9,13}$ The cell-free extracts were incubated with L-His and excess SAM. After the reaction, the product was analyzed by LC-ESI-MS. A specific peak with the same retention time and mass spectrometry (MS) spectrum as the HER standard was clearly detected after $1 \mathrm{~h}$ of incubation, demonstrating that EgtD converted L-His into HER (Figure S3 of the Supporting Information).

We next overproduced recombinant EgtB using pQE1a-Red (Figure S1 of the Supporting Information) and changed the probable start codon TTG into ATG. However, recombinant EgtB formed inclusion bodies in multiple culture conditions. Therefore, a plasmid from which a recombinant enzyme is produced as a maltose binding protein (MBP)-fused enzyme was examined. We successfully produced a MBP-fused EgtB in a soluble form in transformants harboring pQE1a-mMsB. To confirm whether the recombinant enzyme had the expected activity, we carried out in vitro experiments with purified recombinant enzyme (Figure S4 of the Supporting Information). 9 The recombinant MBP-fused EgtB was incubated with HER and $\gamma \mathrm{GC}$ for $2 \mathrm{~h}$. As Figure S5 of the Supporting Information shows, HER completely disappeared and the formation of a new product with $\mathrm{m} / z 462.20$ was detected. On the basis of HR-ESI-FT-MS analysis of the purified compound, the molecular formula of the product was determined to be $\mathrm{C}_{17} \mathrm{H}_{27} \mathrm{O}_{8} \mathrm{~N}_{5} \mathrm{~S}\left(\mathrm{~m} / z\right.$ : $[\mathrm{M}+\mathrm{H}]^{+}$calculated for $\mathrm{C}_{17} \mathrm{H}_{28} \mathrm{O}_{8} \mathrm{~N}_{5} \mathrm{~S}^{+}, 462.16531$; observed, 462.165 07), which corresponded to that of $\gamma \mathrm{GC}$-HER, suggesting the formation of 
(a)

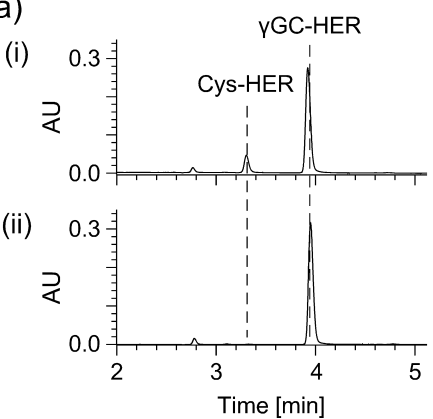

(b)

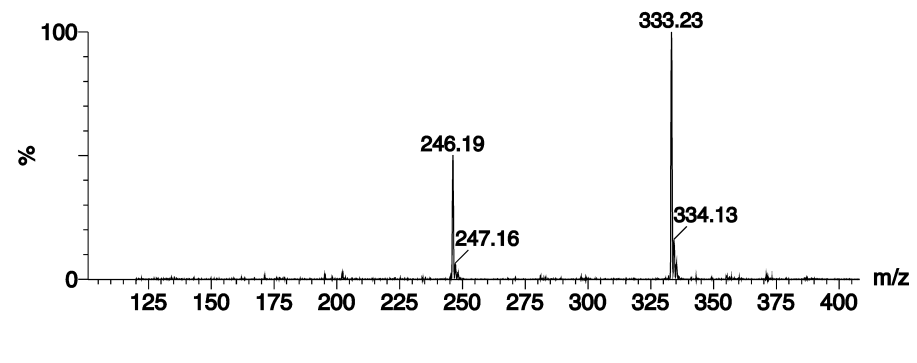

Figure 3. LC-ESI-MS analysis of EgtC reaction products. (a) Traces at $250 \mathrm{~nm}$ of reaction products. The reaction $(40 \mu \mathrm{L})$ was carried out by adding (i) purified recombinant EgtC $(3.9 \mu \mathrm{M})$ or (ii) boiled EgtC to the EgtB reaction solution at $25{ }^{\circ} \mathrm{C}$ for $17 \mathrm{~h}$. (b) MS spectrum of the EgtC reaction product (ESI positive mode).

(a)

(i)

(ii)

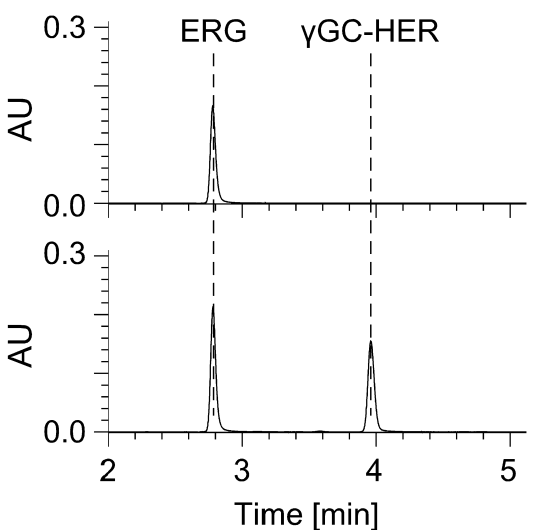

(b)

(i)

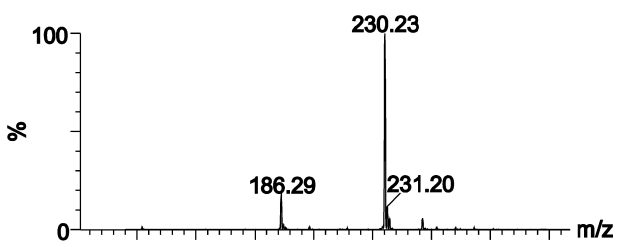

(ii)

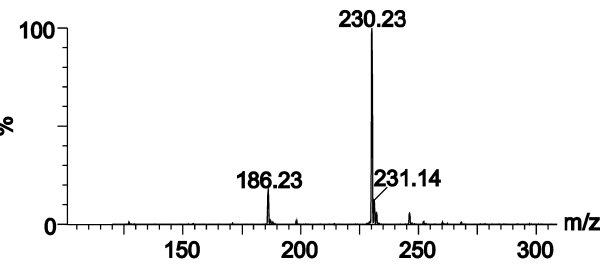

Figure 4. LC-ESI-MS analysis of EgtE reaction products. (a) Traces at $250 \mathrm{~nm}$ of (i) ERG standard and (ii) reaction products. The reaction (40 $\mu \mathrm{L})$ was carried out by adding purified recombinant $\operatorname{EgtE}(2.5 \mu \mathrm{M})$ and $\operatorname{EgtC}(3.9 \mu \mathrm{M})$ (ii) to the boiled supernatant of the EgtB reaction mixture containing $2 \mathrm{mM}$ dithiothreitol at $25{ }^{\circ} \mathrm{C}$ for $17 \mathrm{~h}$. (b) MS spectra of (i) ERG standard and (ii) EgtE reaction product (ESI positive mode).

$\gamma \mathrm{GC}$-HER. The thus formed intermediate compound $\gamma \mathrm{GC}$ HER was purified by high-performance liquid chromatography (HPLC) (Supplementary Methods 2 of the Supporting Information) and used as a standard for quantitative analysis.

Finally, overproduction of recombinant EgtC and EgtE was examined. We first used the same vector as for EgtB production, but no production of either enzyme was observed. We then examined a plasmid from which a recombinant enzyme is produced as a His-tagged enzyme. In this case, both EgtC and EgtE were successfully overproduced. To confirm whether the recombinant enzymes had the expected activity, we carried out in vitro experiments with the purified recombinant enzymes (Figure S4 of the Supporting Information). ${ }^{9}$ Recombinant EgtC was incubated with enzymatically prepared $\gamma$ GC-HER. After $17 \mathrm{~h}$ of reaction, the product was analyzed by LC-ESI-MS. As shown in Figure 3, a new peak with $\mathrm{m} / \mathrm{z}$ 333.23 was detected and Cys-HER formation was suggested. On the basis of HR-ESI-FT-MS analysis of the purified compound, the molecular formula of the product was determined to be $\mathrm{C}_{12} \mathrm{H}_{20} \mathrm{O}_{5} \mathrm{~N}_{4} \mathrm{~S}\left(\mathrm{~m} / \mathrm{z}:[\mathrm{M}+\mathrm{H}]^{+}\right.$calculated for $\mathrm{C}_{12} \mathrm{H}_{21} \mathrm{O}_{5} \mathrm{~N}_{4} \mathrm{~S}^{+}, 333.12272$; observed, 333.122 90), which corresponded to that of Cys-HER. The thus formed Cys-HER was purified (Supplementary Methods 2 of the Supporting Information) and used as a standard for quantitative analysis.

By adding recombinant EgtE together with EgtC into the EgtB reaction mixture, we confirmed the formation of ERG by
LC-ESI-MS analysis (Figure 4), showing that all of the recombinant Egt enzymes possessed the expected activities.

Simultaneous Production of Egt Enzymes for ERG Production. To reconstruct the ERG-producing pathway in $E$. coli, we optimized the production conditions in a stepwise manner: HER, then $\gamma \mathrm{GC}-\mathrm{HER}$, and finally ERG production (Figures 1 and 2).

The first step was HER production. E. coli BW25113 harboring pCF1s-MsD (named strain ET1) was cultured in M9Y medium, and $91 \pm 2 \mathrm{mg} / \mathrm{L}$ HER $(460 \pm 10 \mu \mathrm{M})$ was produced in the culture broth after $48 \mathrm{~h}$. We then carried out feeding experiments with L-His and L-Met, because amino acid biosynthesis in E. coli is strictly regulated by feedback inhibition and/or transcriptional repression. L-Met and L-His feeding increased the yield: $164 \pm 3 \mathrm{mg} / \mathrm{L}$ HER $(833 \pm 15 \mu \mathrm{M})$ was produced after $48 \mathrm{~h}$ of cultivation (Figure 5). After this, L-His and L-Met feeding was employed in all in vivo production experiments.

We next carried out in vivo co-production of EgtD and EgtB for $\gamma$ GC-HER production. Transformants of E. coli BW25113 carrying both pCF1s-MsD and $\mathrm{pQE} 1 \mathrm{a}-\mathrm{mMsB}$ (strain ET2) were cultivated in the medium supplemented with $\mathrm{L}$-His and $\mathrm{L}$ Met. As Figure 6 shows, we confirmed $24 \pm 1 \mathrm{mg} / \mathrm{L} \gamma$ GC-HER $(52 \pm 2 \mu \mathrm{M})$ production after $24 \mathrm{~h}$ of cultivation, indicating that EgtB converted HER to $\gamma \mathrm{GC}$-HER using endogenous $\gamma \mathrm{GC}$ in E. coli. However, accumulation of 3-fold higher amounts of HER $(110 \pm 5 \mathrm{mg} / \mathrm{L})$ than Cys-HER $(36 \pm 2 \mathrm{mg} / \mathrm{L})$ was 


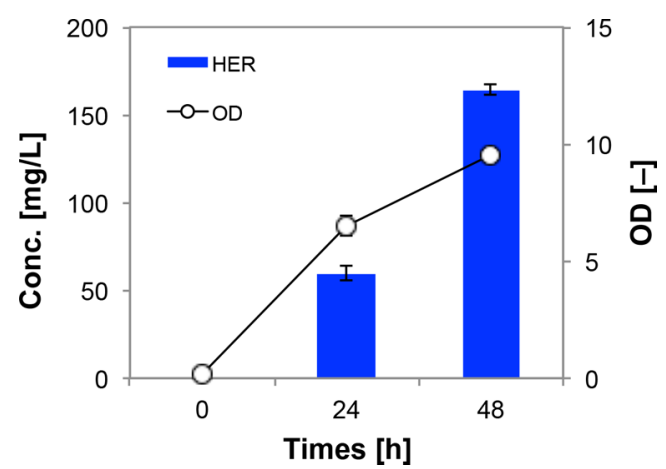

Figure 5. Culture profiles of strain ET1. E. coli BW25113 harboring pCF1s-MsD (strain ET1) was cultured in M9Y medium supplemented with L-His and L-Met. After $3 \mathrm{~h}$ of cultivation, $0.5 \mathrm{mM}$ IPTG was added to the medium. Data are presented as mean values with standard errors from three independent experiments.

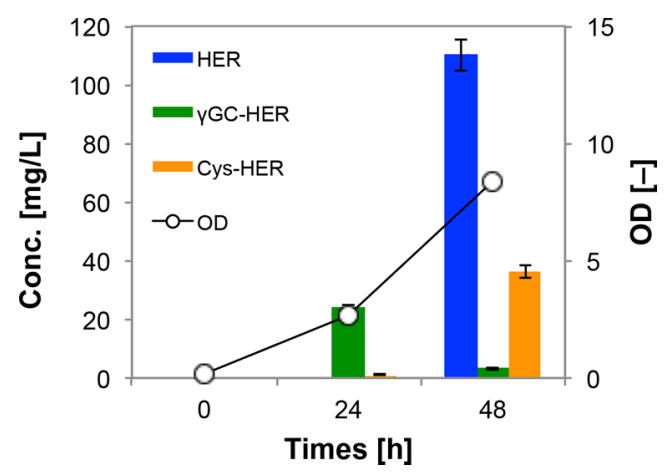

Figure 6. Culture profiles of strain ET2. E. coli BW25113 harboring pCF1s-MsD and pQE1a-mMsB (strain ET2) was cultured in M9Y medium supplemented with L-His and L-Met. After $3 \mathrm{~h}$ of cultivation, $0.5 \mathrm{mM}$ IPTG was added to the medium. Data are presented as mean values with standard errors from three independent experiments.

detected, suggesting that $\gamma$ GC-HER production was ratelimiting.

For simultaneous expression of all egt genes, egtC and egtE, both of which were expressed from the tac promoter of pQE1a, were recloned into plasmid pACYCDuet-1, which is compatible with pQE1a-Red and pCF1s-Red, to construct pAC1c-hMsC/ hMsE (Figure 2 and Supplementary Methods 2 of the Supporting Information). The plasmid was successfully constructed, and production of both enzymes in soluble forms in E. coli was confirmed by SDS-PAGE (Figure S6 of the Supporting Information). The plasmid was introduced into strain ET2 to construct strain ET3, and recombinant enzyme production was examined. As Figure S7 of the Supporting Information shows, all of the enzymes were produced in soluble forms. We then examined ERG production. Strain ET3 produced $19 \pm 2 \mathrm{mg} / \mathrm{L}(83 \pm 8 \mu \mathrm{M})$ ERG together with 73 $\pm 15 \mathrm{mg} / \mathrm{L}(370 \pm 76 \mu \mathrm{M})$ HER in the culture broth after $72 \mathrm{~h}$ of cultivation (Figure 7 and Table 3 ). These results suggested that the EgtB-catalyzed reaction is a bottleneck.

ERG Production and Improvement of Rate-Limiting Steps. Considering that EgtB was overproduced in a soluble form in the producing strain ET3 and that it showed enough activity in in vitro experiments, we considered that insufficient supply of $\gamma \mathrm{GC}$, the substrate of EgtB, might cause the accumulation of HER. To test this hypothesis, overproduction of $\gamma \mathrm{GC}$ synthetase was carried out. To produce $\gamma \mathrm{GC}$ synthetase, the gshA gene from $E$. coli ${ }^{12}$ was cloned and

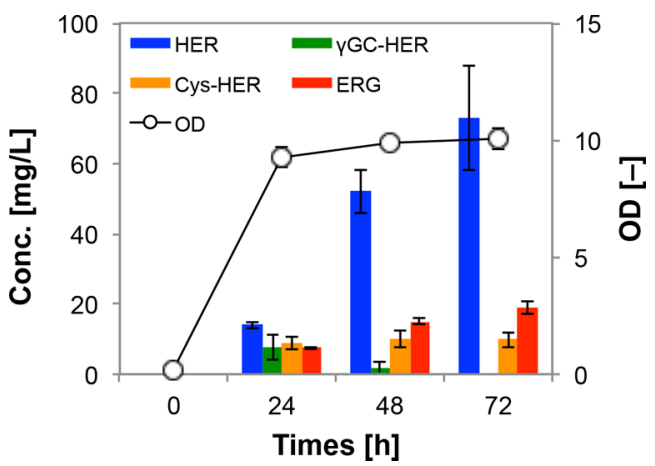

Figure 7. Culture profiles of strain ET3. E. coli BW25113 harboring pCF1s-MsD, pQE1a-mMsB, and pAC1c-hMsC/hMsE (strain ET3) was cultured in M9Y medium supplemented with L-His and L-Met. After $3 \mathrm{~h}$ of cultivation, $0.5 \mathrm{mM}$ IPTG was added to the medium. Data are presented as mean values with standard errors from three independent experiments.

Table 3. Culture Profiles of ET3 and ET4 Strains ${ }^{a}$

\begin{tabular}{lrcccc} 
strain & \multicolumn{1}{c}{ OD } & $\begin{array}{c}\text { HER } \\
(\mathrm{mg} / \mathrm{L})\end{array}$ & $\begin{array}{c}\gamma \mathrm{GC}-\mathrm{HER} \\
(\mathrm{mg} / \mathrm{L})\end{array}$ & $\begin{array}{c}\text { Cys-HER } \\
(\mathrm{mg} / \mathrm{L})\end{array}$ & $\begin{array}{c}\mathrm{ERG} \\
(\mathrm{mg} / \mathrm{L})\end{array}$ \\
$\mathrm{ET3}^{b}$ & $10.1 \pm 0.5$ & $73 \pm 15$ & $\mathrm{ND}^{c}$ & $10 \pm 2$ & $19 \pm 2$ \\
ET4 $^{b}$ & $8.7 \pm 0.2$ & $121 \pm 12$ & $1 \pm 1$ & $9 \pm 1$ & $17 \pm 1$ \\
ET3 $^{d}$ & $11.1 \pm 0.5$ & $48 \pm 17$ & $\mathrm{ND}$ & $9 \pm 0$ & $24 \pm 4$
\end{tabular}

${ }^{a}$ Data after $72 \mathrm{~h}$ of cultivation are presented as mean values with standard error from three independent experiments. ${ }^{b}$ ET3 and ET4 were cultured in M9Y media supplemented with L-His and L-Met. ${ }^{c} \mathrm{ND}$ $=$ not detected. ${ }^{d}$ ET3 was cultured in M9Y media supplemented with L-His, L-Met, and thiosulfate.

inserted into pQE1a-mMsB to construct pQE1a-mMsB/EcA. Although MBP-fused EgtB and GshA were produced in soluble forms in E. coli BW25113 harboring the three plasmids (strain ET4) (Figure S8 of the Supporting Information), ERG productivity was decreased in comparison to that of strain ET3 to $17 \pm 1 \mathrm{mg} / \mathrm{L}(72 \pm 2 \mu \mathrm{M})$ after $72 \mathrm{~h}$ of cultivation (Table 3). In comparison of SDS-PAGE data for strains ET3 and ET4 (Figures S7 and S8 of the Supporting Information), soluble MBP-fused EgtB was found to be decreased in strain ET4. This could be the reason for the reduced productivity.

We then employed another strategy to enhance the $\gamma \mathrm{GC}$ supply. In ERG biosynthesis, $\gamma \mathrm{GC}$ is used as a sulfur donor. In particular, L-Cys is a net sulfur donor, because the L-Glu moiety of $\gamma \mathrm{GC}$ is released by EgtC. Therefore, reinforcement of L-Cys flux may be effective in enhancing $\gamma \mathrm{GC}$ flux and ERG production. Because L-Cys addition into media was reported to be toxic to E. coli cells, ${ }^{14}$ we employed another strategy. We have been studying a L-Cys biosynthetic pathway in E. coli and demonstrated that thiosulfate $\left(\mathrm{S}_{2} \mathrm{O}_{3}{ }^{2-}\right)$ was a better sulfur source than sulfate $\left(\mathrm{SO}_{4}{ }^{2-}\right)$ for high L-Cys production. ${ }^{10} \mathrm{We}$ therefore fed thiosulfate into the growth media. When strain ET3 was cultured in M9Y supplemented with L-Met, L-His, and thiosulfate, ERG productivity was increased to $24 \pm 4 \mathrm{mg} / \mathrm{L}$ $(104 \pm 17 \mu \mathrm{M})$ (Table 3), indicating that reinforcement of LCys flux was very effective in enhancing the ERG production.

In conclusion, to establish a reliable and practical method for ERG supply, fermentative ERG production by microorganisms was investigated. We heterologously overexpressed genes egtBCDE of $M$. smegmatis in $E$. coli and succeeded in the production of ERG $(19 \mathrm{mg} / \mathrm{L})$. Reinforcement of the $\gamma \mathrm{GC}$ supply by feeding of thiosulfate, a suitable sulfur donor for high $\mathrm{L}-\mathrm{Cys}$ production, resulted in higher ERG production $(24 \mathrm{mg}$ / 
L). Considering that the reported ERG contents of mushrooms were from 0.15 to $7.27 \mathrm{mg} / \mathrm{g}$ of dry weight, ${ }^{7}$ our system might become an alternative method for ERG supply. However, significant amounts of HER still accumulated, suggesting that more supply of $\gamma \mathrm{GC}$ and L-Cys by metabolic engineering and use of another EgtB with higher activity are indispensable for high-level production of ERG.

\section{ASSOCIATED CONTENT}

\section{S Supporting Information}

The Supporting Information is available free of charge on the ACS Publications website at DOI: 10.1021/acs.jafc.7b04924.

Supplementary Methods 1 and 2, vectors pQE1a-Red and pCF1s-Red (Figure S1), SDS-PAGE analysis of EgtD production (Figure S2), LC-ESI-MS analysis of EgtD reaction products (Figure S3), SDS-PAGE analysis of purified recombinant EgtB, EgtC, and EgtE (Figure S4), LC-ESI-MS analysis of EgtB reaction products (Figure S5), SDS-PAGE analysis of recombinant EgtC and EgtE production (Figure S6), SDSPAGE analysis of production of recombinant Egt enzymes in strain ET3 (Figure S7), SDS-PAGE analysis of production of recombinant Egt enzymes and GshA in strain ET4 (Figure S8), and primers used in this study (Table S1) (PDF)

\section{AUTHOR INFORMATION}

\section{Corresponding Authors}

*Telephone: +81-11-706-7815. Fax. +81-11-706-7118. E-mail: syasu@eng.hokudai.ac.jp.

*Telephone: +81-11-706-7815. Fax. +81-11-706-7118. E-mail: dairi@eng.hokudai.ac.jp.

\section{ORCID}

Yasuharu Satoh: 0000-0001-6671-7758

Tohru Dairi: 0000-0002-3406-7970

\section{Funding}

This work was supported by the Science and Technology Research Promotion Program for Agriculture, Forestry, Fisheries and Food Industry (26027AB) from Ministry of Agriculture, Forestry and Fisheries (MAFF), Japan (to Iwao Ohtsu).

\section{Notes}

The authors declare no competing financial interest.

\section{ACKNOWLEDGMENTS}

The authors are grateful to Professor Tsutomu Sato (Graduate School of Science and Technology, Niigata University, Niigata, Japan) for providing M. smegmatis genomic DNA. The authors thank James Allen from Edanz Group (www.edanzediting.com) for editing a draft of this manuscript.

\section{NOMENCLATURE}

$\mathrm{ERG}=$ ergothioneine

HER = hercynine

$\gamma \mathrm{GC}=\gamma$-glutamylcysteine

$\gamma$ GC-HER $=$ hercynyl- $\gamma$-glutamylcysteine sulfoxide

Cys-HER = hercynylcysteine sulfoxide

SAM $=S$-adenosylmethionine

$\mathrm{SAH}=S$-adenosylhomocysteine

L-His $=$ L-histidine

L-Glu = L-glutamate
L-Cys $=$ L-cysteine

L-Met = L-methionine

HR-ESI-FT-MS = high-resolution electrospray ionization Fourier transform mass spectrometry

MBP = maltose-binding protein

SDS-PAGE = sodium dodecyl sulfate polyacrylamide gel electrophoresis

\section{REFERENCES}

(1) Genghof, D. S. Biosynthesis of ergothioneine and hercynine by fungi and Actinomycetales. J. Bacteriol. 1970, 103, 475-478.

(2) Pfeiffer, C.; Bauer, T.; Surek, B.; Schömig, E.; Gründemann, D. Cyanobacteria produce high levels of ergothioneine. Food Chem. 2011, 129, 1766-1769.

(3) Pluskal, T.; Ueno, M.; Yanagida, M. Genetic and metabolomic dissection of the ergothioneine and selenoneine biosynthetic pathway in the fission yeast, $S$. pombe, and construction of an overproduction system. PLoS One 2014, 9, e97774.

(4) Gründemann, D.; Harlfinger, S.; Golz, S.; Geerts, A.; Lazar, A.; Berkels, R.; Jung, N.; Rubbert, A.; Schömig, E. Discovery of the ergothioneine transporter. Proc. Natl. Acad. Sci. U. S. A. 2005, 102, $5256-5261$

(5) Cheah, I. K.; Halliwell, B. Ergothioneine; antioxidant potential, physiological function and role in disease. Biochim. Biophys. Acta, Mol. Basis Dis. 2012, 1822, 784-793.

(6) Aruoma, O. I.; Coles, L. S.; Landes, B.; Repine, J. E. Functional benefits of ergothioneine and fruit- and vegetable-derived nutraceuticals: Overview of the supplemental issue contents. Prev. Med. 2012, 54, S4-S8.

(7) Kalaras, M. D.; Richie, J. P.; Calcagnotto, A.; Beelman, R. B. Mushrooms: A rich source of the antioxidants ergothioneine and glutathione. Food Chem. 2017, 233, 429-433.

(8) Genghof, D. S.; Van Damme, O. Biosynthesis of ergothioneine and hercynine by mycobacteria. J. Bacteriol. 1964, 87, 852-862.

(9) Seebeck, F. P. In vitro reconstitution of Mycobacterial ergothioneine biosynthesis. J. Am. Chem. Soc. 2010, 132, 6632-6633.

(10) Kawano, Y.; Onishi, F.; Shiroyama, M.; Miura, M.; Tanaka, N.; Oshiro, S.; Nonaka, G.; Nakanishi, T.; Ohtsu, I. Improved fermentative L-cysteine overproduction by enhancing a newly identified thiosulfate assimilation pathway in Escherichia coli. Appl. Microbiol. Biotechnol. 2017, 101, 6879-6889.

(11) Jones, G. W.; Doyle, S.; Fitzpatrick, D. A. The evolutionary history of the genes involved in the biosynthesis of the antioxidant ergothioneine. Gene 2014, 549, 161-170.

(12) Watanabe, K.; Yamano, Y.; Murata, K.; Kimura, A. The nucleotide sequence of the gene for $\gamma$-glutamylcysteine synthetase of Escherichia coli. Nucleic Acids Res. 1986, 14, 4393-4400.

(13) Vit, A.; Misson, L.; Blankenfeldt, W.; Seebeck, F. P. Ergothioneine biosynthetic methyltransferase EgtD reveals the structural basis of aromatic amino acid betaine biosynthesis. ChemBioChem 2015, 16, 119-125.

(14) Kari, C.; Nagy, Z.; Kovács, P.; Hernádi, F. Mechanism of the growth inhibitory effect of cysteine on Escherichia coli. J. Gen. Microbiol. 1971, 68, 349-356. 